\title{
DETECTION OF MBL PRODUCTION IN PSEUDOMONAS AERUGINOSA IN KATIHAR DISTRICT, BIHAR
}

\author{
Tarannum Yasmin 1 , Krishan Nandan², Aninda Sen ${ }^{3}$
}

${ }^{1}$ Associate Professor, Department of Microbiology, Katihar Medical College, Bihar.

${ }^{2}$ Associate Professor, Department of Microbiology, Katihar Medical College, Bihar.

${ }^{3}$ Professor, Department of Microbiology, Katihar Medical College, Bihar.

\section{ABSTRACT}

\section{BACKGROUND}

Pseudomonas aeruginosa is a leading cause of nosocomial infection due to its numerous intrinsic and acquired mechanisms of drug resistance. Although antibiotic resistance in P. aeruginosa is caused by multiple mechanisms, one major factor leading to resistance is the production of carbapenemases. Metallo- $\beta$-lactamases (MBLs) are carbapenemases which require zinc at the active site and are predominantly produced by $P$. aeruginosa. The selection criteria for confirmation of MBL producers are reduced susceptibility or resistance to carbapenems and/or ceftazidime.

The aim of the study was to determine the efficacy of three antibiotics viz., meropenem, imipenem and ceftazidime as a screening and confirmatory agent for detection of MBL and to assess the diagnostic potential of DDST, DPT and MIC reduction test for detection of MBL.

\section{MATERIALS AND METHODS}

This study included 461 isolates of $P$. aeruginosa collected over a period of 2 years. Resistance to carbapenems and ceftazidime was used for screening of isolates. Positively screened isolates were further subjected to different MBL detecting tests- DDST (Double Disc Synergy Test), DPT (Disc Potential Test) and EPM/EPI Microdilution test.

\section{RESULTS}

A total of 12304 samples were processed during the study period of which 461 isolates were Pseudomonas aeruginosa. Out of 461 Pseudomonas aeruginosa, 45.78\% were MBL producers. Majority of MBL strains were isolated from Department of Surgery (41.23\%). MIC was positive in 211 isolates, being a gold standard test for MBL detection with 100\% sensitivity. DPT was positive in 89.57\% isolates and DDST was positive in 74.4\% isolates. Most MBL strains (93.83\%) were sensitive to Polymyxin B.

\section{CONCLUSION}

The present study gives us an indication regarding the occurrence of MBL production amongst Pseudomonas aeruginosa in Eastern Bihar which is very high at $45.78 \%$. Early detection of MBL producing Pseudomonas aeruginosa may help in appropriate antimicrobial therapy and avoid the development and dissemination of these strains. The DPT was found to be a simple, reliable and reproducible test that showed $89.5 \%$ conformity. Therefore, we recommend incorporation of DPT in all laboratories as a part of routine.

\section{KEYWORDS}

MBL, Pseudomonas Aeruginosa, Carbapenem.

HOW TO CITE THIS ARTICLE: Yasmin T, Nandan K, Sen A. Detection of MBL production in Pseudomonas aeruginosa in Katihar district, Bihar. J. Evolution Med. Dent. Sci. 2017;6(55):4138-4141, DOI: 10.14260/Jemds/2017/896

\section{BACKGROUND}

Pseudomonas aeruginosa ( $P$. aeruginosa) is a leading cause of nosocomial infection due to its numerous intrinsic and acquired mechanisms of drug resistance.(1) Pseudomonas infection is a major cause of concern for treating physicians. Although antibiotic resistance in Pseudomonas aeruginosa is caused by multiple mechanisms, one major factor leading to resistance is the production of carbapenemases.

Pseudomonas aeruginosa producing MBLs were first reported from Japan in 1991 and since then have been reported from various parts of the world, including Asia,

Financial or Other, Competing Interest: None.

Submission 05-06-2017, Peer Review 28-06-2017,

Acceptance 05-07-2017, Published 10-07-2017.

Corresponding Author:

Dr. Tarannum Yasmin,

Department of Microbiology,

Katihar Medical College,

Katihar-854105, Bihar.

E-mail:drtyasmin@gmail.com

DOI: $10.14260 /$ jemds $/ 2017 / 896$
Europe, Australia, South America and North America.(2,3) MBL producing Pseudomonas aeruginosa isolates have been responsible for serious infections, treatment failure and several nosocomial outbreaks in different parts of the world resulting in high morbidity and mortality, increased economic burden and an urgent need to establish a strong infection control protocol.

Metallo- $\beta$-lactamases (MBLs) are carbapenemases which require zinc at the active site and are predominantly produced by P. aeruginosa. (4) They belong to Ambler's class B and Bush-Jacoby Medeiros Group 3 and hydrolyse virtually all $\beta$-lactam agents, including the carbapenems. Further, as the genes coding them are carried on highly mobile elements, their spread in recent years from $P$. aeruginosa to Enterobacteriaceae, has led to a situation where a clinical scenario comparable to the global spread of extended spectrum $\beta$-lactamases is developing. Till now seven main types of MBLs have been described throughout the world IMP, VIM, SPM, GIM, SIM, AIM-1(4) and NDM-1.(5) Among them, bla with worldwide distribution.(6) From India only blavim ${ }^{(7,8)}$ and 
NDM-1(9) have been reported in P. aeruginosa in the past. VIM (Veronese Imipenemase) enzymes have been grouped into three main clusters designated VIM-1, VIM-2 and VIM-7. To date, VIM-2 is more widely spread among P. aeruginosa isolates, whereas VIM-1 is normally confined to Enterobacteriaceae isolates.(10) Clinical and Laboratory Standards Institute (CLSI) has not laid down any specific guidelines though there are several screening methods recommended for detection and confirmation of MBL production in $P$. aeruginosa. The selection criteria for confirmation of MBL producers are reduced susceptibility or resistance to carbapenems and/or ceftazidime. Currently, the most widely accepted standardised MBL confirmation method is the MBL Etest (AB Biodisk, Solna, Sweden). However, due to the high cost many clinical microbiology laboratories use alternative methods such as the double-disc synergy test (DDST) and the combined disc (CD)/disc potentiation test (DPT). Although the DDST and the DPT assay are simple to perform and cheaper than the Etest they have shown discordant results, depending on the employed methodology, $\beta$-lactam substrates, MBL inhibitors (IMBL), and bacterial genus tested.(11) As zinc ion is essential for the action of MBLs, chelating agent like Ethylenediaminetetraacetic acid (EDTA) has been used commonly as in vitro inhibitors of MBLs.

This study was conducted to determine the efficacy of three antibiotics viz., meropenem, imipenem and ceftazidime as a screening and confirmatory agent for detection of MBL and also to assess the diagnostic potential of DDST, DPT and MIC reduction test for detection of MBL.

\section{MATERIALS AND METHODS}

The prospective study was conducted over a period of 2 years from July 2013 to May 2015 in the Department of Microbiology, Katihar Medical College \& Hospital, Katihar, Bihar, India. Patients of both sexes and all age groups were included in this study after obtaining Institutional Ethical Committee clearance.

This study included various clinical samples such as urine, pus, blood and body fluid received for culture. Pseudomonas isolated from stool samples were excluded due to their presence as commensal.

All the specimens collected were subjected to direct microscopy, growth on culture media and a series of tests for identification of Pseudomonas aeruginosa. After identification, these isolates were subjected to antibiotic susceptibility testing by Kirby-Bauer disk diffusion technique according to Clinical and Laboratory Standards Institute (CLSI) guidelines. All the disks were procured commercially (Hi-Media Laboratories Limited, Mumbai, India). The diameter of the zone of inhibition was measured and interpreted according to the CLSI guidelines.

\section{Screening for MBLs}

Isolates of $P$. aeruginosa were considered screen-test positive for MBL if it showed resistance to Imipenem $10 \mu \mathrm{g}$ (IPM) and/or Meropenem $10 \mu \mathrm{g}$ (MEM) and/or Ceftazidime $30 \mu \mathrm{g}$ (CAZ) (HiMedia). Antibiotic sensitivity test was performed by Kirby-Bauer disc diffusion method as per CLSI guidelines.(12)
All positively screened probable MBL producing isolates were subjected to

\section{Double Disc Synergy Test (DDST)}

This test was performed as described by Lee et al(13) with some modifications. IPM $(10 \mu \mathrm{g})$, MEM $(10 \mu \mathrm{g})$, CAZ $(30 \mu \mathrm{g})$ discs were placed on the Muller Hinton agar (MHA) plates seeded with $0.5 \mathrm{McF}$ arland $\left(10^{8} \mathrm{CFU} / \mathrm{mL}\right)$ of test organism with centre to centre distance of $20 \mathrm{~mm}$ from a disc containing $5 \mu \mathrm{L} 0.5 \mathrm{M}$ EDTA $(930 \mu \mathrm{g})$. Plate should be incubated at $37^{\circ} \mathrm{C}$ for $18-20$ hours. Enhancement of zone of inhibition around IPM and/or MEM and/or CAZ toward the EDTA disc in comparison with the zone of inhibition on the far side of corresponding antibiotic disc was interpreted as positive for MBL production. Pseudomonas aeruginosa ATCC 27853 was used as control.

\section{Combined Disc Test/Disc Potentiation Test (DPT)}

This test was performed as described by Yong D et al.(14) Two discs each of $10 \mu \mathrm{g}$ IPM, $10 \mu \mathrm{g}$ MEM and $30 \mu \mathrm{g}$ CAZ placed on lawn culture of 0.5 McFarland test organism seeded on MHA. To one disk of IPM, MEM and CAZ $5 \mu \mathrm{L}$ of 0.5 M EDTA $(930$ $\mu \mathrm{g})$ was added. Plates were incubated at $37^{\circ} \mathrm{C}$ for $18-20$ hours. After incubation, zone of inhibition was measured using Hi-media scale. Organisms which showed increased zone of inhibition by $7 \mathrm{~mm}$ or more (DPT-1: Yong D et al) ${ }^{(14)}$ around any or all of the three discs with EDTA or showed increase by $5-28 \mathrm{~mm}$ inhibition around only CAZ-EDTA disc (DPT-2: Hemlatha et al)(15) as compared to IPM, MEM and CAZ discs alone, respectively, considered to be MBL producers. A blank disc of EDTA was tested as control.

\section{EPM/EPI Microdilution Test: EDTA Phenanthroline + MEM/IPM Microdilution Test(16)}

MIC was determined in 96 well microtitre plates using $50 \mu \mathrm{L}$ of Muller Hinton broth, $5 \mu \mathrm{L}$ of bacterial inoculums $\left(5 \times 10^{4}\right.$ CFU: Soon after preparation) and $50 \mu \mathrm{L}$ of corresponding concentration of the antibiotic per well as per CLSI guidelines.(10) IPM/MEM concentration in the range of 0.25 $512 \mu \mathrm{g} / \mathrm{mL}$ was tested. Reduction in MIC of IPM/MEM will be determined by adding $5-\mu \mathrm{L}$ mixture of chelators EDTA 0.4 $\mathrm{mM}$ and 1, 10-phenanthroline $0.04 \mathrm{mM}$ to the second row, just before inoculation of wells with broth culture. Growth controls without IPM/MEM but with and without chelators mixture were included. Results were recorded by visual inspection of microtitre plates after 18 hours of incubation at $37^{\circ} \mathrm{C}$ as per CLSI guidelines. ${ }^{(10)}$ The test was considered valid when acceptable growth (More or equal to $2 \mathrm{~mm}$ button or definite turbidity) occurred in the positive control well. Absence of turbidity or buttons of less than $2 \mathrm{~mm}$ diameter in the test well were thus taken as the MIC of the organism under test. $\mathrm{A} \geq$ four-fold IPM and/or MEM MIC reduction in presence of chelators as compared to MIC without them was taken as cut-off value for MBL production.

\section{RESULTS}

A total of 12304 samples were received in the laboratory in which 7261 showed no growth. 5043 samples showed growth of different bacterial isolates. 1426/5043 (28.27\%) showed growth of GPC in pure culture, 2876/5043 (57.02\%) showed growth of GNB in pure culture, 449/5043 (8.90\%) 
showed mixed growth and 292/5043 (5.79\%) showed presence of candida species.

Amongst 2876 GNB isolates, only 461/2876 (16.02\%) showed the growth of Pseudomonas aeruginosa which were processed for final study. Out of 461 Pseudomonas aeruginosa, 211/461 (45.78\%) were MBL producers and 250/461 (54.22\%) were non-MBL producers.

Out of 211 MBL strains, $118 / 211$ (55.92\%) were isolated from male patients and 93/211 (44.08\%) were isolated from female patients. Maximum number of MBL producers were seen in age group of $21-30$ years (36.97\%) followed by the age group of 31 - 40 years (20.38\%).

Maximum number of cases were MBL producers which were isolated from pus samples 149/211 (70.61\%), followed by urine samples $59 / 211$ (27.96\%), blood $2 / 211(0.94 \%)$ and body fluid $1 / 211(0.47 \%)$.

Majority of MBL strains were isolated from Dept. of Surgery $87 / 211$ (41.23\%) followed by Dept. of Orthopaedics 42/211 (19.90\%), Dept. of Medicine 39/211 (18.48\%), Dept. of Obstetrics \& Gynaecology 36/211 (17.06\%) and Dept. of Paediatrics 7/211 (3.31\%) [Table: 1].

Based on the screening criteria for MBL production, 211 isolates were screened positive. The tests for MBL detection were performed on 211 positively screened isolates. MIC was positive in 211 isolates, being a gold standard test for MBL detection with $100 \%$ sensitivity. DPT was positive in 189 $(89.57 \%)$ isolates and DDST was positive in 157 (74.4\%) isolates. In this study, MIC showed the highest sensitivity (100\%) while DDST was the least sensitive test $(74.4 \%)$ [Table: 2].

Among 211 MBL strains, only 198/211 (93.83\%) were sensitive to Polymyxin B, 183/211 (86.72\%) were sensitive to Colistin. Nitrofurantoin $41 / 59(69.49 \%)$ was sensitive against urinary isolates only.

\begin{tabular}{|c|c|c|c|c|c|}
\hline \multirow{2}{*}{ Department } & \multicolumn{2}{|c|}{ MBL } & \multicolumn{2}{c|}{ Non-MBL } & \multirow{2}{*}{ Total } \\
\cline { 2 - 5 } & IPD & OPD & IPD & OPD & \\
\hline Medicine & 18 & 21 & 23 & 31 & 93 \\
\hline Surgery & 59 & 28 & 24 & 39 & 150 \\
\hline Orthopaedics & 24 & 18 & 21 & 36 & 99 \\
\hline OBG & 17 & 19 & 22 & 33 & 91 \\
\hline Paediatrics & 04 & 03 & 07 & 14 & 28 \\
\hline Total & 122 & 89 & 97 & 153 & 461 \\
\hline Table 1. Department wise Distribution of \\
MBL and Non-MBL Producers \\
\hline
\end{tabular}

\begin{tabular}{|c|c|c|}
\hline Name of Test & Positive & Percentage \\
\hline MIC & 211 & 100 \\
\hline DPT & 189 & 89.57 \\
\hline DDST & 157 & 74.4 \\
\hline \multicolumn{2}{|c|}{ Table 2. Comparative Study of Different Methods } \\
for MBL Detection (n=211) \\
\hline
\end{tabular}

\section{DISCUSSION}

MBL production is a significant problem in hospital isolates of $P$. aeruginosa. With increasing isolation of ESBL-producing isolates in the hospital setting necessitating the use of carbapenems, the problem of MBL production is also increasing. (8) A case controlled study from Japan showed that patients infected with MBL-producing $P$. aeruginosa were more likely to receive multiple antibiotics and also infection related deaths due to MBL-producing $P$. aeruginosa were more frequent than the death caused by MBL negative $P$. aeruginosa.(17)

In this study, out of $461 P$. aeruginosa isolates, 211 isolates were screened out as probable MBL producers based on their resistance to carbapenems and CAZ. We found $24.6 \%$ resistance among $P$. aeruginosa to IPM and meropenem each while $29.8 \%$ resistance to CAZ. A study done at a tertiary care hospital in Puducherry (India) in 2006 reported 10.9\% resistance to Carbapenems, (18) while another study in Puducherry in 2008 reported $31.1 \%$ resistance to meropenem.(19) A 5-year longitudinal study from Latin America has reported that $P$. aeruginosa resistance to carbapenems has risen to $40 \%$.(20) These findings show there is a rising trend in the carbapenem resistance among the $P$. aeruginosa.

Phenotypic tests for MBL have not been nationally or internationally standardised. PCR analysis is the gold standard method for detection of MBL producers, but it is not suitable for daily testing in clinical laboratories due to the cost and inconvenience. (21)

In our study, DPT and DDST also showed good sensitivity for MBL detection as $89.5 \%$ and $74.4 \%$ respectively. DPT is a rapid and easy performed test, hence we found the DPT to be satisfactory for detecting MBL as it is an easy procedure and is simple to interpret in accordance with other studies. ${ }^{(14,22,23)}$ Similar to our observation, Behera et al(1) and $\mathrm{Qu}$ et al (11) reported that DPT is better than DDST for routine MBL detection.

\section{CONCLUSION}

The present study gives us an indication regarding the occurrence of MBL production amongst Pseudomonas aeruginosa in Eastern Bihar. Unfortunately, the number isolated by us is alarmingly large $(45.78 \%)$. It has also been observed that majority of these MBL producers were from indoor patients which indicates that most of these strains are hospital acquired and the presence of these organisms in the hospital environment is a man-made phenomenon due to overuse and misuse of broad spectrum antibiotics. Proper infection control practices and formulation of a hospital antibiotic usage policy is clearly indicated.

The other main issue that needs to be addressed is early detection of MBL producing Pseudomonas aeruginosa which will help in appropriate antimicrobial therapy and avoid the development and dissemination of these strains. The DPT was found to be a simple, reliable and reproducible test that showed $89.5 \%$ conformity with the MIC reduction test which is time taking, difficult to interpret and needs a high degree of precision. It is recommended that DPT be incorporated in all laboratories as a part of routine antibiotic susceptibility testing procedures especially on strains showing resistance to any one of the carbapenems.

\section{REFERENCES}

[1] Behera B, Mathur P, Das A, et al. An evaluation of four different phenotypic techniques for detection of metallo-beta-lactamase producing pseudomonas aeruginosa. Indian J Med Microbiol 2008;26(3):233-7.

[2] Watanabe $M$, Iyobe $S$, Inoue $M$, et al. Transferable imipenem resistance in pseudomonas aeruginosa. Antimicrob Agents Chemother 1991;35(1):147-51. 
[3] Pitout JD, Gregson DB, Poirel L, et al. Detection of pseudomonas aeruginosa producing metallo-betalactamases in a large centralized laboratory. J Clin Microbiol 2005;43(7):3129-35.

[4] Gupta V. Metallo beta lactamases in pseudomonas aeruginosa and Acinetobacter species. Expert Opin Investig Drugs 2008;17(2):131-43.

[5] Struelens MJ, Monnet DL, Magiorakos AP, et al. New Delhi metallo-beta-lactamase 1-producing Enterobacteriaceae: emergence and response in Europe. Euro Surveill 2010;15(46):19716.

[6] Kouda S, Ohara M, Onodera $M$, et al. Increased prevalence and clonal dissemination of multidrugresistant pseudomonas aeruginosa with the blaIMP-1 gene cassette in Hiroshima. J Antimicrob Chemother 2009;64(1):46-51.

[7] Castanheira M, Bell JM, Turnidge JD, et al. Carbapenem resistance among pseudomonas aeruginosa strains from India: evidence for nationwide endemicity of multiple metallo-beta-lactamase clones (VIM-2,-5,-6 and -11 and the newly characterized VIM-18). Antimicrob Agents Chemother 2009;53(3):1225-7.

[8] Manoharan A, Chartterjee S, Mathai D. Detection and characterization of metallo beta lactamases producing pseudomonas aeruginosa. Indian J Med Microbiol 2010;28(3):241-4.

[9] Dugal S, Fernandes A. Carbapenem hydrolysing metallo- $\beta$-lactamases: a review. Int J Curr Pharma Res 2011;3(3):9-16.

[10] Walsh TR, Toleman MA, Poirel L, et al. Metallo-betalactamases: the quiet before the storm? Clin Microbiol Rev 2005;18(2):306-25.

[11] Qu TT, Zhang JL, Wang J, et al. Evaluation of phenotypic tests for detection of metallo-\{beta\}lactamases-producing pseudomonas aeruginosa strains in China. J Clin Microbiol 2009;47(4):1136-42.

[12] Clinical and laboratory standards institute. Methods for dilution antimicrobial susceptibility tests for bacteria that grow aerobically, 8th ed. Approved standard M07-A8. Wayne, PA 2009:P 29.

[13] Lee K, Lim YS, Yong D, et al. Evaluation of the Hodge test and the imipenem-EDTA double-disk synergy test for differentiating metallo-\{beta\}-lactamaseproducing isolates of pseudomonas spp. and Acinetobacter spp. J Clin Microbiol 2003;41:4623-9.
[14] Yong D, Lee $\mathrm{K}$, Yum JH, et al. Imipenem-EDTA disk method for differentiation of metallo-beta-lactamasesproducing clinical isolates of pseudomonas spp and Acinetobacter spp. J Clin Microbiol 2002;40(10):3798801.

[15] Hemalatha V, Sekar U, Kamat V. Detection of metallo beta lactamase producing pseudomonas aeruginosa in hospitalized patients. Indian J Med Res 2005;122(2):148-52.

[16] Migliavacca R, Docquire JD, Mugnaioli C, et al. Simple microdilution test for detection of metallo-betalactamase production in pseudomonas aeruginosa. J Clin Microbiol 2002;40(11):4388-90.

[17] Saderi H, Karimi Z, Owlia P, et al. Phenotypic detection of metallo-beta-lactamase producing pseudomonas aeruginosa strains isolated from burned patients. Iran J Pathol 2008;3(1):20-4.

[18] Kanungo R, Srinivasan S, Devi S. Emerging resistance to carbapenems in hospital acquired pseudomonas infection: a cause for concern. Indian J Pharmacol 2006;38(4):287-8.

[19] Noyal MJ, Menezes GA, Harish BN, et al. Simple screening tests for detection of carbapenemases in clinical isolates of nonfermentative gram-negative bacteria. Indian J Med Res 2009;129(6):707-12.

[20] Andrade SS, Jones RN, Gales AC, et al. Increasing prevalence of antimicrobial resistance among pseudomonas aeruginosa isolates in Latin American medical centres: 5 year report of the SENTRY antimicrobial surveillance program (1997-2001). J Antimicrob Chemother 2003;52(1):140-1.

[21] Galani I, Rekatsina PD, Hatzaki D, et al. Evaluation of different laboratory tests for the detection of metallobeta-lactamase production in Enterobacteriaceae. J Antimicrob Chemother 2008;61(3):548-53.

[22] Berges L, Rodriguez-Villalobos H, Deplano A, et al. Prospective evaluation of imipenem/EDTA combined disc and etest for detection of metallo-beta-lactamaseproducing pseudomonas aeruginosa. J Antimicrob Chemother 2007;59(4):812-3.

[23] Yan JJ, Wu JJ, Tsai SH, et al. Comparison of the doubledisk, combined disk, and etest methods for detecting metallo-beta-lactamases in gram-negative bacilli. Diagn Microbial Infect Dis 2004;49(1):5-11. 